\title{
Iron limitation by transferrin promotes simultaneous cheating of pyoverdine and exoprotease in
} Pseudomonas aeruginosa

Oswaldo Tostado-Islas ${ }^{1+}$, Alberto Mendoza-Ortiz ${ }^{1+}$, Gabriel Ramírez-García ${ }^{1+}$, Isamu Daniel CabreraTakane $^{1+}$, Daniel Loarca ${ }^{1+}$, Caleb Pérez-González ${ }^{1+}$, Ricardo Jasso-Chavez ${ }^{2}$, J Guillermo JiménezCortés $^{1}$, Yuki Hoshiko ${ }^{3}$, Toshinari Maeda ${ }^{3}$, Adrian Cazares ${ }^{4,5}$, Rodolfo García-Contreras ${ }^{1 *}$

1. Departamento de Microbiología y Parasitología, Facultad de Medicina, Universidad Nacional Autónoma de México, Mexico City, Mexico.

2. Departamento de Bioquímica, Instituto Nacional de Cardiología, Mexico City, Mexico.

3. Department of Biological Functions Engineering, Kyushu Institute of Technology, Kitakyushu, Japan.

4. EMBL's European Bioinformatics Institute (EMBL-EBI), Wellcome Genome Campus, Hinxton, Cambridge, United Kingdom

5. Wellcome Sanger Institute, Wellcome Genome Campus, Hinxton, Cambridge, United Kingdom

+ These authors contributed equally

*Correspondence: R García-Contreras, Departamento de Microbiología y Parasitología, Facultad de Medicina, Universidad Nacional Autónoma de México, 04510, Mexico City, Mexico.E mail: rgarc@bq.unam.mx

*Adrian Cazares,_EMBL's European Bioinformatics Institute (EMBL-EBI), Wellcome Genome Campus, Hinxton, Cambridge, United Kingdom. E mail: acaza@ebi.ac.uk

\section{Abstract}

Pseudomonas aeruginosa is the main bacterial model to study cooperative behaviors, since it yields exoproducts such as exoproteases and siderophores that act as public goods and can be exploited by selfish non-producers that behave as social cheaters. Non-producers of the siderophore pyoverdine are typically isolated in media with low free iron, mainly casamino acids medium supplemented with transferrin. Nevertheless, using a protein as the iron chelator could 
additionally select mutants unable to produce exoproteases that degrade the transferrin to facilitate iron release. Here, we investigated the dynamics of pyoverdine and exoprotease production in media in which iron was limited by using either transferrin or a cation chelating resin. Our experiments show that concomitant loss of pyoverdine and exoprotease production readily develops in media with transferrin whereas only lack of pyoverdine emerges in medium treated with the resin. Genomic characterization of the exoprotease- and pyoverdine-less mutants revealed large deletions (13 to $33 \mathrm{~Kb}$ ) including Quorum Sensing (lasR, rsal and lasl) and flagellar genes. Complementation experiments, PCR and motility tests confirmed the deletions. Our work shows that using transferrin as an iron chelator imposes simultaneous selective pressure for the loss of pyoverdine and exoprotease production. The unintended effect of transferrin observed in our experiment settings can help revisiting or informing the design of similar studies.

Keywords: pyoverdine, public goods, apo-transferrin, social cheating, exoprotease, genomic deletions

\section{Introduction}

Pseudomonas aeruginosa is a major bacterial model to study cooperative behaviors since it produces a variety of exoproducts, such as siderophores, which are considered public goods [1]. These molecules are important iron scavenging agents, essential for iron acquisition in iron-limited media; however, its production is susceptible to exploitation by social cheaters, since siderophores are released to the environment and can benefit both producer and non-producer individuals. Most of the published works aiming to study siderophore cheating in $P$. aeruginosa have used casamino acids (CAA) medium supplemented with $100 \mu \mathrm{g} / \mathrm{mL}$ of human apo-transferrin (CAA TF) in order to chelate iron and make iron acquisition and growth dependent on siderophore production[2-7]. This medium was originally proposed by Griffin and coworkers in 2004. In 2017 Harrison and collaborators compiled a list of published experiments on siderophore cheating in $P$.

57 aeruginosa to that date, covering 23 articles and 36 experiments, of which 34 were performed in 58 CAA TF [2]. These works include studies on the long-term coevolution between cheaters (PAO1 $59 \quad p v d D)$ and cooperators (PAO1 wild-type) pyoverdine cheating and cheating resistance in isolates 60 from soil and ponds and the dynamics of exoprotease and pyoverdine non-producers in media 61 with protein as sole carbon source and under iron limitation[8]. 
More recently, the use of the CAA TF medium has been reported in research addressing the role of

63 transposable phages in the evolution of social strategies in iron-limiting medium [9], the effect of

64 cheating in diversity of pyoverdine variants and the effect of nutrients availability in the dynamics

65 between wild-type and non-siderophore-producing mutants [4].

66 The CAA TF medium has been used assuming it creates conditions specifically suitable for the 67 selection of non-siderophore producers, nevertheless, it is well known that quorum sensing (QS)68 dependent exoproteases such as elastase LasB and alkaline protease AprA greatly enhance the 69 rate of iron acquisition through pyoverdine both in vitro and in vivo [10,11]. Hence, likely during 70 infections, iron acquisition may depend on both siderophore production and exoproteases 71 activity. Exoproteases are also public goods, and therefore susceptible of exploitation by non72 producers [12-16]. In most studies of pyoverdine dynamics, iron in the medium has been limited by the action of apotransferrin, a protein susceptible to degradation by $P$. aeruginosa QS-regulated exoproteases; hence, we hypothesized iron acquisition in such conditions is mediated by two types of public goods: siderophores (pyoverdine) and QS-regulated exoproteases that facilitate iron release from the degraded transferrin. Consequently, the production of both pyoverdine and exoproteases would be susceptible to be cheated by non-producers, favoring the selection of these mutants. Here we show that limiting iron availability by transferrin (TF) promotes strong selection of exoprotease non-producers in addition to pyoverdine-less mutants. Our experiments identified

81 different phenotypes emerging from continuous growth in CAA TF medium in terms of pyoverdine and exoprotease production, but double cheaters of these exoproducts were prevalent at the end of the experiments. Whole genome sequencing and analysis of three mutant types allowed the detection of small sequence variants associated to loss of pyoverdine production but also uncovered extensive genome deletions in the exoprotease non-producers. The deletions included QS genes, essential to produce exoproteases, as well as genes encoding diverse functions such as drove the emergence of exoprotease-less individuals through large genomic rearrangements affecting phenotypes other than siderophore production, an unintended effect that might have 90 influenced similar studies.

\section{Methods}




\section{Strains media and growth conditions}

93

94

95

96

97

98

99

100

101

102

103

104

105

106

107

108

109

110

111

112

113

114

115

116

117

118

119

120

The strains used in this work and their characteristics are summarized in Table 1.

Bacteria was cultured in CAA medium, containing per liter: $5 \mathrm{~g}$ casamino acids, $1.18 \mathrm{~g}$ $\mathrm{K}_{2} \mathrm{HPO}_{4} 3 \mathrm{H}_{2} \mathrm{O}, 0.25 \mathrm{~g} \mathrm{MgSO} 4.7 \mathrm{H}_{2} \mathrm{O}$ and $25 \mathrm{mM}$ HEPES buffer, CAA TF medium (CAA medium supplemented with $20 \mathrm{mM} \mathrm{NaHCO}$ and $100 \mathrm{ug} / \mathrm{mL}$ of human apo-transferrin), CAA chelex medium (CAA treated with chelex 100 resin according the manufacturer instructions $15 \mathrm{~g}$ of resin per $100 \mathrm{~mL}$ of medium). Minimal succinate medium (MSM per liter: $6 \mathrm{~g} \mathrm{~K}_{2} \mathrm{HPO}_{4}, 3 \mathrm{~g} \mathrm{KH}_{2} \mathrm{PO}_{4}, 1 \mathrm{~g}$ $\left(\mathrm{NH}_{4}\right)_{2} \mathrm{SO}_{4}, 0.2 \mathrm{~g} \mathrm{MgSO}_{4} \cdot 7 \mathrm{H}_{2} \mathrm{O}$, and $4 \mathrm{~g}$ succinic acid), and MSM TF (MSM supplemented with 20 $\mathrm{mM} \mathrm{NaHCO}_{3}$ and $100 \mathrm{ug} / \mathrm{mL}$ of human apo-transferrin) Final $\mathrm{pH}$ was 7-.0.

Bacteria were cultured in Erlenmeyer flasks of $50 \mathrm{~mL}$ o capacity filled with $5 \mathrm{~mL}$ of medium, at $37^{\circ} \mathrm{C}$ with $200 \mathrm{rpm}$ orbital shaking, during $18 \mathrm{~h}$ for growth experiments and $24 \mathrm{~h}$ for continuous sub-culturing, each new culture was initiated with enough bacteria to reach an O.D. $600 \mathrm{~nm}$ of 0.05 .

\section{Isolation of pyoverdine and exoprotease less individuals}

Single colonies were obtained by streaking each subculture made in a pair day in LB plates that were further incubated at $37^{\circ} \mathrm{C}$ for $18 \mathrm{~h}$, after that $20-50$ colonies were passed to King A plates and were incubated under the same conditions, those that were green and showed fluorescence under UV light were considered pyoverdine producers. Also, colonies were passed to M9 minimal medium plates with casaminoacids $0.025 \%(\mathrm{w} / \mathrm{V})$ and $0.5 \%(\mathrm{w} / \mathrm{V})$ casein and were incubated under the same conditions; those that produced a clear halo (indicating casein degradation) were considered exoprotease producers. Some single colonies exhibiting different phenotypes were picked, grown in liquid CAA medium and stored in $16 \%$ glycerol at $-70{ }^{\circ} \mathrm{C}$ for further experiments.

\section{Bacterial competitions}

In order to evaluate the cheating capacity of some of the isolated colonies that were nonproducers of pyoverdine and or non-producers of exoprotease, they were incubated in CAA, CAA TF and M9 Casein medium in co-culture with the PA14 wild-type strain at $15-20 \%$ as an initial amount and incubated during $24 \mathrm{~h}$, samples were taken just after inoculation and at $24 \mathrm{~h}$ of growth and colonies were isolated for phenotypic determination (pyoverdine and exoprotease production) in order to calculate the initial and final proportions. 


\section{Iron determination}

122 The content of $\mathrm{Fe}$ in growth media was determined by digesting $5 \mathrm{~mL}$ of each one with $5 \mathrm{~mL}$ of

123 concentrated nitric acid with overnight incubation at $90^{\circ} \mathrm{C}$. The content of metals was determined

124 by atomic absorbance spectrometry (ASS) by using a Varian apparatus as reported elsewhere.

125 Pyoverdine, exoprotease and pyocyanin measurements

126 Pyoverdine from the bacterial supernatants was measured fluorometrically, using a Perkin Elmer

127 Victor Nivo plate reader, exciting at $405 \mathrm{~nm}$ and recording at $510 \mathrm{~nm}$.

128 The exoprotease activity found in the supernatants was determined by measuring hydrolysis of

129 Azo-casein (SIGMA, St Louis Mo, USA) as described in [17], using also a Perkin Elmer Victor Nivo 130 plate reader determining absorbance at $415 \mathrm{~nm}$.

131 Pyocyanin was extracted from the supernatants using chloroform, re extracted with HCL 0.2 N and 132 determined spectrophotometrically as previously described [18].

\section{Swimming motility assays}

134 Swimming plates were made with $0.25 \%$ (W/V) agar Luria broth (LB). After solidification, plates 135 were briefly dried at room temperature. A spot inoculated with $2.5 \mu \mathrm{L}$ aliquots taken directly from 136 overnight LB cultures. Swim plates were incubated at $37^{\circ} \mathrm{C}$ for $18-20 \mathrm{~h}$. An image of the swimming 137 plate was captured with a digital camera.

\section{Whole genome sequencing and variant calling analysis}

139 Genomic DNA used for sequencing was extracted from overnight cultures. Library synthesis was 140 performed using the Nextera XT DNA Sample Prep Kit (Illumina, San Diego CA, USA) according to 141 the manufacturer's instructions. TruSeq HT adapters (Illumina, San Diego CA, USA) were used to 142 barcode the libraries, and each library was sequenced using an Illumina MiSeq 300 bp paired-end 143 instrument. Trimmomatic v.0.39 [19] was used to process the raw fastq files for adapters and 144 quality trimming with the following settings: "LEADING:3, TRAILING:3, SLIDINGWINDOW:4:23 145 MINLEN:35".

146 Single nucleotide polymorphisms (SNPs) and small indels were detected with the Snippy v3.2 147 pipeline (https://github.com/tseemann/snippy) by aligning the sequencing reads to the genome of 
the reference strain UCBPP-PA14 (Accession: NC_008463.1) with BWA-MEM (Li, H. Aligning sequence reads, clone sequences and assembly contigs with BWA-MEM, calling variants with FreeBayes [20] and annotating them with snpEffm [21]. The coverage metrics of the genes las $R$ and pvdS were determined with samtools v.1.7 [22]. Deletions flanking lasR were inferred from coverage distribution data obtained with BEDTools [23] as the longest stretch of gene coding regions featuring zero reads. De novo assembly of the genomes was carried out using SPAdes v.3.10.1 [24] with default settings. Visualization of coverage distribution and inspection of the joining regions was performed with Artermis [25]. The genome assemblies are publicly available from the BioProject PRJNA540579.

\section{Complementation of the strains}

The construction pUCP20-lasR was donated by Professor Gloria Soberón Chavez from the Institute of Biomedical Research at UNAM, for the pUCP20-pvdS construction, pvdS gene was cloned by amplifying it from the PA14 genomic DNA, standard methods were used to isolate chromosomal DNA [26]. Restriction DNA enzymes and T4 DNA ligase were purchased from England Biolabs (NEB, Ipswich MA, USA) or Invitrogen. Plasmid used for cloning the sigma factor $p v d S$ (was purified using an E.Z.N.A Plasmid mini kit I, (Q-spin) (Omega Bio-Tek). DNA was amplified with the appropriate oligonucleotides using Phusion High-Fidelity DNA polymerase (Thermo scientific, Sweden) according to the manufacturer's recommendations. The resulted pvdS obtained by PCR was purified by E.Z.N.A. Cycle pure kit (Omega Bio-tek). pUCP20-pvdS plasmid carries a 742pb fragment that includes the coding region of $p v d S$ (564 pb), $151 \mathrm{pb}$ upstream and 27pb downstream. For this, pvdS was amplified by PCR using the oligonucleotides pvdS-Fw: 5'GCTCTAGAGCCAGCATGCGGACCATTCAC3' and pvdS-Rv: 5'GCGAATTCACCGGCGCTGAGGAATGC3' The product was then cloned into pUCP20 as an Xbal-EcoRI fragment, the resultant plasmid pUCP20-pvdS, was inserted by transformation into $P$. aeruginosa. Complementation of the las $R$ less phenotype was done in the presence and absence of $5 \mu \mathrm{M} 3-\mathrm{O} \times 0-\mathrm{C}_{12}-\mathrm{HSL}$ (Sigma) and pyoverdine, caseinolytic activity and pyocyanin was determined as explained previously.

\section{Transformation of Pseudomonas aeruginosa by $\mathrm{CaCl}_{2}$}

Briefly, P. aeruginosa was grown overnight in Luria Broth (LB) medium with orbital shaking (200 $\mathrm{rpm}$ ) at $37^{\circ} \mathrm{C} .250 \mu \mathrm{L}$ of the cells culture were harvested by centrifugation at $15000 \times \mathrm{g} 1 \mathrm{~min}$, washed in same volume of cold sterile Milli-Q water. Then the cell suspension was centrifuged at 
$15000 \times \mathrm{g} 1 \mathrm{~min}$ and the supernatant was discarded. The cell pellet was suspended in $250 \mu \mathrm{L}$ of $0.1 \mathrm{mM} \mathrm{CaCl}_{2}$ and incubated for $20 \mathrm{~min}$ at $4^{\circ} \mathrm{C}$. The cells were centrifuged at $15000 \times \mathrm{g} 1 \mathrm{~min}$. The supernatant was retired and the cell pellet suspended in $100 \mu \mathrm{l}$ of $0.1 \mathrm{mM} \mathrm{CaCl}_{2}$ whit $100 \mathrm{ng}$ of the pUCP20-pvdS construction or the empty plasmid. The suspension was maintained at $4^{\circ} \mathrm{C}$ for $1 \mathrm{~h}$. Cells were heat pulsed by partially immersing in water at $42^{\circ} \mathrm{C}$ for $2 \mathrm{~min}$. After the heat step the cells suspension was incubated at $4^{\circ} \mathrm{C}$ for $2 \mathrm{~min}$. $500 \mu \mathrm{L}$ of LB medium was added to the cells and then incubated at $37^{\circ} \mathrm{C}$ for $1 \mathrm{~h}$. Transformant colonies were selected on King-B plates [27] containing $300 \mu \mathrm{g} / \mathrm{mL}$ carbenicillin.

\section{PCRs for the confirmation of deletions}

The following PCR reactions were done in order to confirm the presence of genomic deletions: amplification of a fragment of $742 \mathrm{pb} b p$ of the gene $p v d S$ as positive control, using the primers pvdS-Fw 5'GCTCTAGAGCCAGCATGCGGACCATTCAC $\quad 3^{\prime}$ and pvdS-Rv 5'GCGAATTCACCGGCGCTGAGGAATGC 3', to confirm the integrity of the chromosomal DNA isolated from the wild-type $P$. aeruginosa as well as the mutants $\mathrm{CH} 1, \mathrm{CH} 2$ and $\mathrm{CH} 3, \mathrm{~b}$ ) fragment of $720 \mathrm{bp}$ of the gene lasR, using the primers F- lasR 5'ATGGCCTTGGTTGACGGTT 3' and R-lasR 5'GCAAGATCAGAGAGTAATAAGACCCA 3'. All the PCR reactions was done like previously described.

\section{Statistical analysis}

Results shown are the average of at least 3 independent experiments \pm SD or SEM, data was analyzed using the IBM SPSS Statistics software and the statistical tests: Mann-Whitney, KruskallWallis, and Student T-test were applied to different data sets as specified in the figure legends, differences were considered significant when P values were lower than 0.05 .

\section{Results}

\section{Selection of siderophore and exoprotease non-producers in the presence of apo-transferrin}

Colonies of the $P$. aeruginosa strain PA14 were grown and serially transferred in CAA medium supplemented with apo-transferrin (CAA TF) for 14 serial passages and periodically tested for pyoverdine and exoproteases production. Siderophore-less mutants were identified from passage number 6 ( $\sim 24$ generations). Figure $1 \mathrm{~A}$, shows that in parallel to the selection of pyoverdine-less mutants, there was a strong selection of exoprotease non-producers. A screening of single 
207

208

209

210

211

212

213

214

215

216

217

218

219

220

221

222

223

224

225

226

227

228

229

230

231

232

233

234

235

236

colonies of the two phenotypes revealed that most of them exhibit concomitant loss of pyoverdine and exoproteases production (Figure S1).

To test if the presence of apo-transferrin was responsible for the selection of exoprotease-less mutants, a new set of experiments was done in the CAA medium using the chelex 100 resin as the iron-depletion factor. Non-treated CAA and minimal succinate medium (MSM) were also used as a control and as an alternative of iron-limited medium, respectively. Our results show that pyoverdine non-producers appeared from passage 6 (Figure $1 \mathrm{~A}$ and $\mathrm{B}$ ) and were selected upon passage 14 in all media; however, they were more abundant in the CAA medium treated with chelex, and in MMS, than in the non-treated CAA medium (Figure 1B), as expected from the lower iron availability in those media $(13.1 \pm 3.25,7.12 \pm 1.8$, and $66.3 \pm 24.7 \mu \mathrm{M}$ for CAA chelex, MMS, and CAA respectively). We did not find exoprotease non-producers in the screening of bacteria grown in media lacking apo-transferrin (Figure 1B). In contrast, adding apo-transferrin to the MSM promoted the selection of exoprotease-less mutants in parallel to pyoverdine non-producers (Figure 1B), thus confirming that the presence of apo-transferrin imposes a selective pressure for the loss of exoprotease production. The addition of TF to the CAA medium also promoted the emergence of exoprotease- and pyoverdine-less individuals in sequential cultures of the reference strain PA01 (Figure S2), thus suggesting that this selection may not be exclusive to the strain PA14.

\section{Characterization of pyoverdine and exoprotease non-producer individuals}

Our experiments demonstrated that the CAA TF medium selects the loss of both pyoverdine and exopreotease production, hence, four different types of individuals are expected to occur in the population: wild-type (wt, pyoverdine and exoprotease producers), pyoverdine cheaters (pyoverdine non-producer, exoprotease producers), exoprotease cheaters (pyoverdine producer, exoprotease non-producers), and double cheaters (pyoverdine and exoprotease non-producers). The presence of the four types of individuals was verified in the experiment in CAA TF medium and their frequency from passage 6 to 14 was recorded. We found that double cheaters were the predominant cheater type from passage 8 and that they reached around $90 \%$ of the population in the passage 14 (Figure S1), hence implying that these individuals have a greater fitness than those losing a single trait.

Next, we characterized a set of individual colonies by measuring production of pyoverdine and exoproteases (caseinolytic activity), and growth in different media. The colonies selected were 
representative of the three different cheater phenotypes: one colony identified as pyoverdine and exoprotease non-producer $(\mathrm{CH} 1)$, other identified as pyoverdine producer but exoprotease nonproducer $(\mathrm{CH} 3)$, and the last colony identified as pyoverdine non-producer but exoprotease producer $(\mathrm{CH} 2)$. We found that the growth of the three isolates was similar to PA14 wild-type in CAA medium; however, the pyoverdine non-producers $(\mathrm{CH} 1$ and $\mathrm{CH} 2)$ grew less than the wild-type strain in iron-limited media (CAA TF and CAA chelex) (Figure S3). Likewise, exoprotease nonproducers ( $\mathrm{CH} 1$ and $\mathrm{CH} 3)$ grew less than the wild-type strain in the medium containing casein as sole carbon source (Figure S3). As expected, the levels of fluorescence in the pyoverdine nonproducers $\mathrm{CH} 1$ and $\mathrm{CH} 2$ were considerably lower than in the $\mathrm{CH} 3$ isolate (Figure $2 \mathrm{~A}$ ). The caseinolytic activity assay also confirmed the reduction in exoprotease activity in $\mathrm{CH} 1$ and $\mathrm{CH} 3$ but not in the $\mathrm{CH} 2$ strain (Figure 2B). Competition experiments between PA14 wild-type and two different $\mathrm{CH}$ strains showed that $\mathrm{CH} 1$ can cheat both pyoverdine and exoprotease since its frequency in co-cultures increased after 24 hours in CAA TF and M9 Casein media but not in nontreated CAA medium (Figure $3 \mathrm{~A}$ ), whilst $\mathrm{CH} 2$ increased its frequency only in an iron-limiting condition (Figure 3B).

\section{Whole genome sequencing}

To get insights into the genetic basis of the loss of exoprotease and pyoverdine production, we performed whole genome sequencing of the PA14 wild-type strain used in this work, the three $\mathrm{CH}$ isolates, and an additional clone with wild-type phenotype isolated from the CAA-TF experiments (WT1). A variant calling analysis targeting small indels and nucleotide polymorphisms identified few mutations occurring in the $\mathrm{CH}$ isolates but not in the wild-type and WT1 strains (Supplementary Table 1), nevertheless, their association with the observed phenotypes was not entirely clear. Unlike previous reports $[3,9,14,15,28]$, we did not detect mutations in the pvdS and lasR genes which are commonly associated with loss of pyoverdine and exoprotease production. The pyoverdine non-producer isolates $\mathrm{CH} 1$ and $\mathrm{CH} 2$, however, featured a frameshift mutation in $p v d A$ and one mutation in the intergenic region upstream the $p v d S$ gene, respectively. To assess whether low sequencing coverage hampered the identification of mutations in the regions encoding LasR and PvdS, we determined the coverage metrics for these genes in all the isolates (Supplementary Table 1). The pvdS sequence was covered by hundreds of reads in all the genomes with comparable RPKM values. In contrast, very few or no reads mapping las $R$ were detected in the genomes of the exoprotease non-producers $\mathrm{CH} 1$ and $\mathrm{CH} 3$. Detailed examination of 
268

269

270

271

272

273

274

275

276

277

278

279

280

281

282

283

284

285

286

287

288

289

290

291

292

293

294

295

296

the lasR-flanking regions in both genomes revealed several genes with zero reads coverage, indicative of large deletions (Figure 4). The coverage assessment of the regions predicted the loss of $\sim 33$ and $13 \mathrm{~kb}$ in the $\mathrm{CH} 1$ and $\mathrm{CH} 3$ genomes, respectively (Figure 4). The putative deletions included flagellar genes, components of an RND multidrug efflux pump, and the quorum sensing genes lasl, rsaL and lasR (entirely or partially) (Supplementary Table 1). Additionally, the deletion in $\mathrm{CH} 1$ covered some transporters, regulators, and a larger number of flagellar genes, among others.

\section{Confirmation of genome deletions and motility tests}

We designed primers targeting the genes las $R$ and $p v d S$ to validate the deletions identified in the $\mathrm{CH} 1$ and $\mathrm{CH} 3$ isolates. The fragment corresponding to the amplification of las $R$ was detected in the strains $\mathrm{CH} 2$ and wild type but not in $\mathrm{CH} 1$ and $\mathrm{CH} 3$ (Figure S4). Conversely, pvdS was amplified in all the strains, as anticipated from our genome analysis. Since deletions in $\mathrm{CH} 1$ and $\mathrm{CH} 3$ include several flagellar genes, we further assessed the functional impact of this genomic loss by evaluating the swimming motility of the isolates. Neither $\mathrm{CH} 1$ nor $\mathrm{CH} 3$ exhibited motility, in contrast to the wild type and $\mathrm{CH} 2$ strains which lack the genome deletion (Figure S5).

Next, we assembled the $\mathrm{CH} 1$ and $\mathrm{CH} 3$ genomes to identify the newly generated joining regions and better define the structure of the deletions. Contigs containing junctions between the regions flanking the deletions were recognized in the two genomes (Figure 5). Detailed examination of the junctions revealed that in $\mathrm{CH} 1$ the deletion removed $33.2 \mathrm{~kb}$ of genome sequence including 30 coding regions and different portions of the genes $f / h B$ and RS18715 where the chromosome breaks occurred. In $\mathrm{CH} 3$, the intergenic region between fliL and fliK, and the gene las $R$, correspond to the chromosome breakpoints of the $13.5 \mathrm{~kb}$ deletion that removed 11 genes and 157 of the 240 codons in lasR. In CH3 the breaks rejoin merging the flhB and RS18715 coding sequences whereas in $\mathrm{CH} 1$ the junction likely leads to loss of the lasR ORF coding potential.

\section{Complementation of cheater phenotypes}

Our genome sequence analysis revealed large deletions in the strains $\mathrm{CH} 1$ and $\mathrm{CH} 3$ encompassing the Quorum-sensing (QS) genes lasI and lasR (partially or entirely). Multiple studies on exoprotease cheating report that non-producers selected in medium containing protein as sole 
carbon source are commonly characterized as lasR-less mutants. Since the isolates carrying genome deletions lack lasl, we hypothesized that loss of exoprotease production could be complemented by an exogenous lasR only with the addition of the $3-0 \times 0-\mathrm{C}_{12}-\mathrm{HSL}$ signal. Consistent with this notion, the transformation of $\mathrm{CH} 3$ with the construction pUCP20-las $R$ led to recover exoprotease production only in the presence of $3-0 \times 0-C_{12}-H S L$ in the medium (Figure S4A). Pyocyanin production, also dependent on functional QS, was determined in the $\mathrm{CH} 3$ strain carrying pUCP20-lasR, with and without the $3-\mathrm{O} \times \mathrm{xo}^{-} \mathrm{C}_{12}-\mathrm{HSL}$ signal. The results showed that $\mathrm{CH} 3$ is deficient in pyocyanin production, which can be complemented with pUCP20-las $R$ only when the signal is added (Figure S6A). Likewise, $\mathrm{CH} 1$ recovered exoprotease production in the presence of pUCP20lasR and 3-Oxo- $\mathrm{C}_{12}-\mathrm{HSL}$ (Figure S6B). We also tested whether the $\mathrm{CH} 2$ isolate (pyoverdine nonproducer, exoprotease producer) was defective due to lack of expression of the sigma factor pvdS, finding that complementation with an exogenous pvdS largely alleviates its deficit in pyoverdine production (Figure S6C).

\section{Discussion}

Siderophore cheating has been extensively studied in the laboratory using $P$. aeruginosa as the main model since it represents an important and frequent behavior in communities of free-living bacteria [29]. Lack of pyoverdine production is also common, and increases with colonization time, in clinical $P$. aeruginosa isolates from cystic fibrosis patients that retain the ability to uptake it hence becoming potential cheaters. Similarly, $P$. aeruginosa QS-deficient mutants unable to produce exoproteases are commonly isolated from infections [30] in coexistence with QSproficient isolates [31]. Loss of public good production and cheating seem to be a frequent phenomenon in $P$. aeruginosa and may contribute to attenuate its virulence during chronic infections; in fact, inoculating social cheaters in infections has been proposed as an strategy to attenuate host damage [32], supported by experimental evidence from animal infection models [33].

The strong selection for loss of exoprotease production upon cultivation with apo-transferrin presented here suggests that iron acquisition in such conditions not only depends on pyoverdine production, as initially assumed, but also relies on the activity of exoproteases that break down the transferrin, facilitating the release of the iron bound to it and enhancing the rate of iron acquisition through pyoverdine [10]. Since both siderophore and exoproteases are public goods, 
327 mutants that loss the expression of one or both traits, are benefited by the production of those

328 factors by the cooperators, thus exploiting them.

329 Our genome analyses revealed that the loss of exoprotease production in the $\mathrm{CH} 1$ and $\mathrm{CH} 3$ 330 mutants is not due to punctual mutations in las $R$ but rather to extensive genome deletions 331 including the las $R$ and lasl genes; accordingly, complementation of exoprotease production in 332 these mutants was only achieved by adding las $R$ in the presence of 3-Oxo- $\mathrm{C}_{12}-\mathrm{HSL}$ (Figure S4 AB). 333 Moreover, there was a cluster of flagellar genes among the genes included in the deletions, 334 consistent with the lack of swimming motility detected in the exoprotease-less individuals. In 335 contrast with the loss of exoproteases in $\mathrm{CH} 1$ and $\mathrm{CH} 3$, loss of pyoverdine in $\mathrm{CH} 2$ was 336 complemented by the addition of $p v d S$, in agreement with a mutation on the putative promoter 337 region of this gene. Similar mutations affecting $p v d S$ have also been found in other studies [3].

338 The lack of motility developed in some of our isolates is in line with previous findings reporting the 339 inactivation of flagellar genes through punctual mutations in both pyoverdine non-producers and 340 cooperators when the PA01 strain is serially cultured in CAA TF [3] and suggests that the lack of 341 motility could be beneficial in such culture conditions. One possible explanation is that swimming 342 does not confer an advantage during growth with shaking but the synthesis and function of the 343 flagellum is energetically costly, therefore, losing it would allow the bacterium to save energy for 344 other processes. Intriguingly, no deletion including lasIR genes was reported in the Kummerli et al. 345 study [3]; we sought assessing the presence of deletions affecting the las/R genes in the genome 346 sequences presented in this work, however, these were not publicly available.

347 Other studies have found mutations in motility genes upon sub-culturing in CAA and CAA TF media 348 [9]. Likewise, the archetypical strain to study siderophore cheating PA06609 harbor mutations in 349 motility genes, mutations in lasl, and a deletion of $6 \mathrm{~kb}$ in a region that includes genes involved 350 with the synthesis of the pyoverdine side chain [2]; hence suggesting that losing QS and likely the 351 ability to make exoproteases may be favorable for cheating in this mutant, and indicating that 352 deletions can also be responsible for loss of pyoverdine production.

353 Experimental evolution studies investigating the development of resistance against the non-redox 354 iron III mimetic gallium also involve the use of apo-transferrin to limit iron in CAA medium. We 355 consider that selection for loss of exoprotease production represents a key variable likely 356 overlooked in the experiments of these studies. In line with our findings, Bonchi and colleagues 
357 [34] reported a weak correlation between pyoverdine production and the ability of $P$. aeruginosa 358 clinical strains to grow in human serum, nevertheless, a strong correlation between growth and 359 exoprotease production was identified. The positive correlation between growth and proteolytic 360 activity was due to degradation of the transferrin present in the serum leading to facilitation of

361 iron release and uptake by pyoverdine, which translated into reduced susceptibility to the 362 inhibitory effects of gallium [34].

363 Recent research has shown that coexistence between wild-type cells and pyoverdine (pvdS 364 mutants) and exoprotease (lasR mutants) non-producers is stable in low-iron conditions with 365 casein as sole carbon source since the medium used in the experiments of this study contains apo366 transferrin for achieving iron limitation[8], it would be interesting to test whether the same 367 stability is observed when the iron availability is limited by methods other than the utilization of 368 iron chelating proteins.

369 While others have shown that CAA TF do not necessarily resemble the growth conditions of $P$. 370 aeruginosa in vivo here we show that strong selection for exoprotease-less mutants during 371 sequential cultures in this medium may have also been overlooked, hence, we propose the 372 utilization of other strategies for generating iron-limiting media. In our case, the use of iron373 chelating resins removed $80 \%$ of the iron in the medium; alternatively, non-protein iron chelators 374 such as 2,2?-bipyridine have been used to study siderophore cheating in Burkholderia cenocepacia 375 [35]. Based on the outcome of our genome analysis, we also encourage authors investigating 376 siderophore cheating to revise their sequencing data seeking for gene deletions that may 377 responsible for important phenotypic changes, including the loss of exoprotease production and 378 motility.

Acknowledgements

381 R G-C research is funded by CONACYT grant CB 2017-2018 number A1-S-8530 and by PAPITT 382 UNAM grant number IN214218, we are grateful with Cecilia Martinez Castillo for her technical 383 assistance with some experiments. 


\section{Figure legends}

Figure 1

388

A) Percentage of pyoverdine and exoprotease non-producers of the PA14 strain PA14 identified during sequential subculture in CAA medium supplemented with transferrin (CAA TF). Results are the average of 8 independent experiments \pm SEM. No significant differences between the percentages of non-pyoverdine and non-protease producers were found using a Mann-Whitney U test.

B) Percentage of pyoverdine (top) and exoproteases (bottom) non-producers of the strain PA14 identified during sequential subculture in CAA medium, CAA medium treated with chelex 100 (CAA ch), minimal succinate medium (MMS) and minimal succinate medium supplemented with transferrin (MMS TF). Results are the average of 3 independent experiments \pm SEM.

\section{Figure 2}

A) Pyoverdine production of the PA14 wild-type strain, 3 PA14-derived isolates from the evolution experiments $(\mathrm{CH} 1, \mathrm{CH} 2, \mathrm{CH} 3)$, and a las $R$ deletion mutant. Plotted are the averages of at least 5 independent experiments \pm SEM. Significant differences in production of pyoverdine between PA14 WT and $\mathrm{CH} 1$ ( $p=0.023)$, PA14 WT and $\mathrm{CH} 2(p=0.001), \mathrm{CH} 2$ and $\mathrm{CH} 3(\mathrm{p}=0.019)$, and PA14 WT and lasR ( $\mathrm{p}=0.005$ ) were found using a Krustall-Wallis test $(\mathrm{p}<0.001)$ with Bonferroni correction.

B) Exoprotease production (caseinolitic activity) of the PA14 wild-type strain, 3 PA14-derived isolates from the evolution experiments $(\mathrm{CH} 1, \mathrm{CH} 2, \mathrm{CH} 3)$, and a las $R$ deletion mutant. The values plotted correspond to the average of at least 5 independent experiments \pm SEM. Significant differences in exoprotease production between PA14 WT and $\mathrm{CH} 1$ ( $p=0.005)$, PA14 WT and $\mathrm{CH} 3$ ( $\mathrm{p}=0.002), \mathrm{CH} 1$ and $\mathrm{CH} 2$ ( $\mathrm{p}=0.015), \mathrm{CH} 2$ and las $(\mathrm{p}=0.007)$, and PA14 WT and las $(\mathrm{p}=0.002)$ were found using a Krustall-Wallis test $(p<0.001)$ with Bonferroni correction.

\section{Figure 3}

Competitions between the PA14 wild-type strain and $\mathrm{CH} 1$ (A) or $\mathrm{CH} 3$ (B) in CAA medium, CAA medium supplemented with transferrin (CAA TF), and caseinate medium (CAS). The barplots show the average \pm SD of the initial (grey bars) and after $24 \mathrm{~h}$ (White bars) proportion of $\mathrm{CH} 1$ in the competition experiments. The experiments were performed in triplicate. The differences between 
414 the initial and after $24 \mathrm{~h}$ proportions of $\mathrm{CH} 1$ in CAA TF and CAS media are significant according to

415 T-test, $\mathrm{P}>0.05$. Difference between proportions of $\mathrm{CH} 3$ are significant in CAA TF medium.

$416 \quad$ Figure 4

417 Multigene deletions on the genomes of the isolates $\mathrm{CH} 1$ and $\mathrm{CH} 2$. The figure shows the coverage 418 distribution of sequencing reads mapping a selected region of the PA14 genome (NC_008463).

419 Coordinates of the selected region are displayed above the sequencing coverage plot. Coverage by 420 reads from the $\mathrm{CH} 1, \mathrm{CH} 2$, or wild-type (WT) genomes is color coded and indicated in the figure. A 421 white dotted line in the plot indicates a reference coverage value. The position and approximate 422 length of regions featuring zero mapped reads from the $\mathrm{CH} 1$ and $\mathrm{CH} 2$ genomes, indicative of 423 deletions, is marked by dotted lines below the coverage plot. A gene map of the PA14 selected 424 region is shown at the bottom of the figure with genes represented by white boxes. Names or 425 locus tags of genes of interest are indicated above or below their corresponding boxes.

\section{$426 \quad$ Figure 5}

427 Junctions between regions flanking multigene deletions in the $\mathrm{CH} 1$ and $\mathrm{CH} 3$ genomes. Diagrams in 428 the figure illustrate the chromosome breaks (top) and joining regions (bottom) of deletions 429 identified in the genomes of the $\mathrm{CH} 1$ (left) and $\mathrm{CH} 3$ (right) isolates. Gene maps in the diagrams are 430 draw to scale according to the annotations and coordinates of the PA14 genome (NC_008463). 431 Genes are represented by white boxes. Gene names or locus tags are shown next to their 432 corresponding boxes. The deletion breakpoints in the chromosome are depicted as zigzag lines in 433 the maps and the genes affected by them are marked in bold typeface. The nucleotide sequences 434 flanking the breakpoints prior deletion (top) and after junction (bottom), are presented in the 435 figure. The position of the breakpoints prior deletion is indicated at the center of the diagrams 436 along with the length of the deletion. The joining regions displayed in the figure were identified in 437 contigs resulting from the assembly of the $\mathrm{CH} 1$ and $\mathrm{CH} 3$ genomes.

\section{References}

$440 \quad$ 1. Smith P, Schuster M. Public goods and cheating in microbes. 2019.

441 2. Harrison F, McNally A, Da Silva AC, Heeb S, Diggle SP. Optimised chronic infection models 
demonstrate that siderophore 'cheating' in Pseudomonas aeruginosa is context specific. ISME J 2017.

3. Kümmerli R, Santorelli LA, Granato ET, Dumas Z, Dobay A, Griffin AS, et al. Co-evolutionary dynamics between public good producers and cheats in the bacterium Pseudomonas aeruginosa. J Evol Biol 2015.

4. Stilwell P, Lowe C, Buckling A. The effect of cheats on siderophore diversity in Pseudomonas aeruginosa. J Evol Biol 2018.

5. Butaite E, Baumgartner M, Wyder S, Kümmerli R. Siderophore cheating and cheating resistance shape competition for iron in soil and freshwater Pseudomonas communities. Nat Commun 2017.

6. Jin Z, Li J, Ni L, Zhang R, Xia A, Jin F. Conditional privatization of a public siderophore enables Pseudomonas aeruginosa to resist cheater invasion. Nat Commun 2018.

7. Leinweber A, Fredrik Inglis R, Kümmerli R. Cheating fosters species co-existence in wellmixed bacterial communities. ISME J 2017.

8. Özkaya Ö, Balbontín R, Gordo I, Xavier KB. Cheating on Cheaters Stabilizes Cooperation in Pseudomonas aeruginosa. Curr Biol 2018.

9. O’Brien S, Kümmerli R, Paterson S, Winstanley C, Brockhurst MA. Transposable temperate phages promote the evolution of divergent social strategies in Pseudomonas aeruginosa populations. Proc R Soc B Biol Sci 2019.

10. Wolz C, Hohloch K, Ocaktan A, Poole K, Evans RW, Rochel N, et al. Iron release from transferrin by pyoverdin and elastase from Pseudomonas aeruginosa. Infect Immun 1994.

11. Kim SJ, Park RY, Kang SM, Choi MH, Kim CM, Shin SH. Pseudomonas aeruginosa alkaline protease can facilitate siderophore-mediated iron-uptake via the proteolytic cleavage of transferrins. Biol Pharm Bull 2006.

12. Sandoz KM, Mitzimberg SM, Schuster M. Social cheating in Pseudomonas aeruginosa quorum sensing. Proc Natl Acad Sci U S A 2007; 104: 15876-15881.

13. Diggle SP, Griffin AS, Campbell GS, West SA. Cooperation and conflict in quorum-sensing 
bacterial populations. Nature 2007; 450: 411-414.

470

471

472

473

474

475

476

477

478

479

480

481

482

483

484

14. Dandekar AA, Chugani S, Greenberg EP. Bacterial quorum sensing and metabolic incentives to cooperate. Science (80- ) 2012; 338: 264-266.

15. Loarca D, Díaz D, Quezada H, Guzmán-Ortiz AL, Rebollar-Ruiz A, Presas AMF, et al. Seeding Public Goods Is Essential for Maintaining Cooperation in Pseudomonas aeruginosa. Front Microbiol 2019; 10.

16. García-Contreras R, Loarca D, Pérez-González C, Jiménez-Cortés JG, Gonzalez-Valdez A, Soberón-Chávez G. Rhamnolipids stabilize quorum sensing mediated cooperation in Pseudomonas aeruginosa. FEMS Microbiol Lett 2020.

17. Loarca D, Díaz D, Quezada H, Guzmán-Ortiz AL, Rebollar-Ruiz A, Presas AMF, et al. Seeding Public Goods Is Essential for Maintaining Cooperation in Pseudomonas aeruginosa. Front Microbiol 2019; 10: 1-8.

18. Castañeda-Tamez P, Ramírez-Peris J, Pérez-Velázquez J, Kuttler C, Jalalimanesh A, SaucedoMora $\mathrm{M}$, et al. Pyocyanin restricts social cheating in Pseudomonas aeruginosa. Front Microbiol 2018; 9: 1-10.

19. Bolger AM, Lohse M, Usadel B. Trimmomatic: A flexible trimmer for Illumina sequence data. Bioinformatics 2014.

20. Garrison E, Marth G. Haplotype-based variant detection from short-read sequencing -- Free bayes -- Variant Calling -- Longranger. arXiv Prepr arXiv12073907 2012.

21. Cingolani P, Platts A, Wang LL, Coon M, Nguyen T, Wang L, et al. A program for annotating and predicting the effects of single nucleotide polymorphisms, SnpEff. Fly (Austin) 2012.

22. Li H, Handsaker B, Wysoker A, Fennell T, Ruan J, Homer N, et al. The Sequence Alignment/Map format and SAMtools. Bioinformatics 2009.

23. Quinlan AR, Hall IM. BEDTools: A flexible suite of utilities for comparing genomic features. Bioinformatics 2010.

24. Bankevich A, Nurk S, Antipov D, Gurevich AA, Dvorkin M, Kulikov AS, et al. SPAdes: A new genome assembly algorithm and its applications to single-cell sequencing. J Comput Biol 
2012.

497 25. Carver T, Harris SR, Berriman M, Parkhill J, McQuillan JA. Artemis: An integrated platform for visualization and analysis of high-throughput sequence-based experimental data.

26. Ausubel FM, Brent R, Kingston RE, Moore DD, Seidman JG, Smith JA, et al. Current Protocols in Molecular Biology: Preface. Curr Protoc Mol Biol . 2010.

27. King EO, Ward MK, Raney DE. Two simple media for the demonstration of pyocyanin and fluorescin. J Lab Clin Med 1954.

28. Sandoz KM, Mitzimberg SM, Schuster M. Social cheating in Pseudomonas aeruginosa quorum sensing. Proc Natl Acad Sci U S A 2007.

506 Bioinformatics 2012.

$$
\text { quorum sensing. Proc Natl Acad Sci U S A } 2007 .
$$

29. D'Onofrio A, Crawford JM, Stewart EJ, Witt K, Gavrish E, Epstein S, et al. Siderophores from Neighboring Organisms Promote the Growth of Uncultured Bacteria. Chem Biol 2010.

30. Wang Y, Gao L, Rao X, Wang J, Yu H, Jiang J, et al. Characterization of lasR-deficient clinical isolates of Pseudomonas aeruginosa. Sci Rep 2018.

31. Wilder CN, Allada G, Schuster M. Instantaneous within-patient diversity of Pseudomonas aeruginosa quorum-sensing populations from cystic fibrosis lung infections. Infect Immun 2009; 77: 5631-5639.

32. Brown SP, West SA, Diggle SP, Griffin AS. Social evolution in micro-organisms and a Trojan horse approach to medical intervention strategies. Philos Trans R Soc B Biol Sci . 2009.

33. Rumbaugh KP, Diggle SP, Watters CM, Ross-Gillespie A, Griffin AS, West SA. Quorum Sensing and the Social Evolution of Bacterial Virulence. Curr Biol 2009.

34. Bonchi C, Frangipani E, Imperi F, Visca P. Pyoverdine and proteases affect the response of Pseudomonas aeruginosa to gallium in human serum. Antimicrob Agents Chemother 2015.

35. Sathe S, Mathew A, Agnoli K, Eberl L, Kümmerli R. Genetic architecture constrains exploitation of siderophore cooperation in the bacterium Burkholderia cenocepacia. Evol Lett 2019. 
bioRxiv preprint doi: https://doi.org/10.1101/2020.06.21.163022; this version posted June 21, 2020. The copyright holder for this preprint (which was not certified by peer review) is the author/funder. All rights reserved. No reuse allowed without permission.

522 

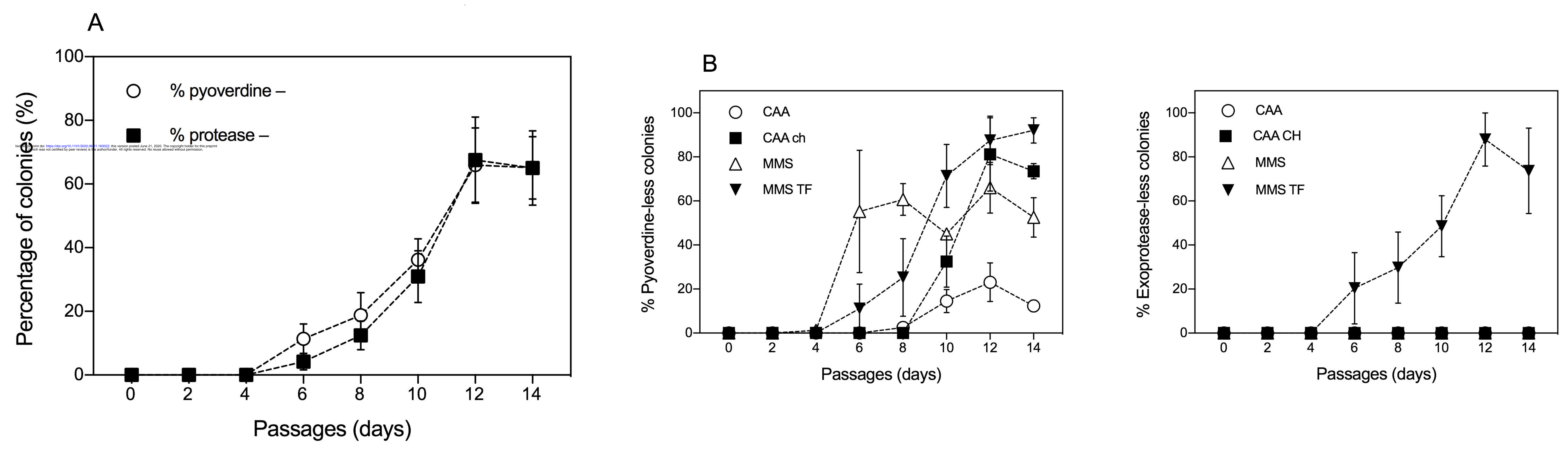

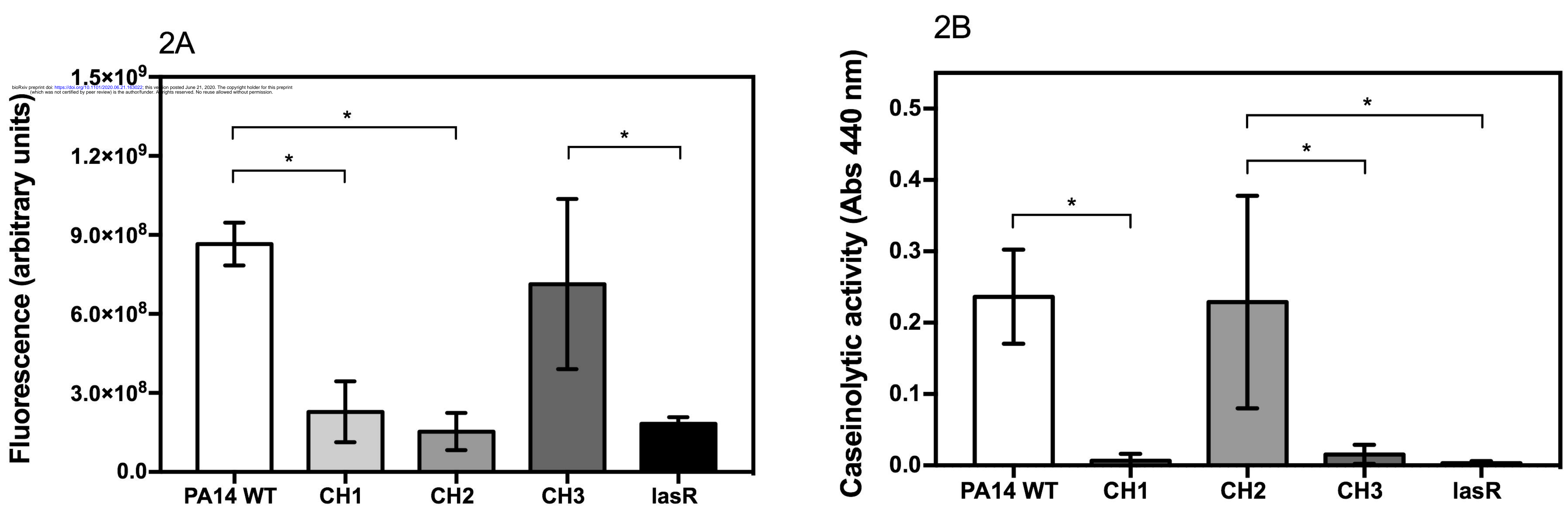

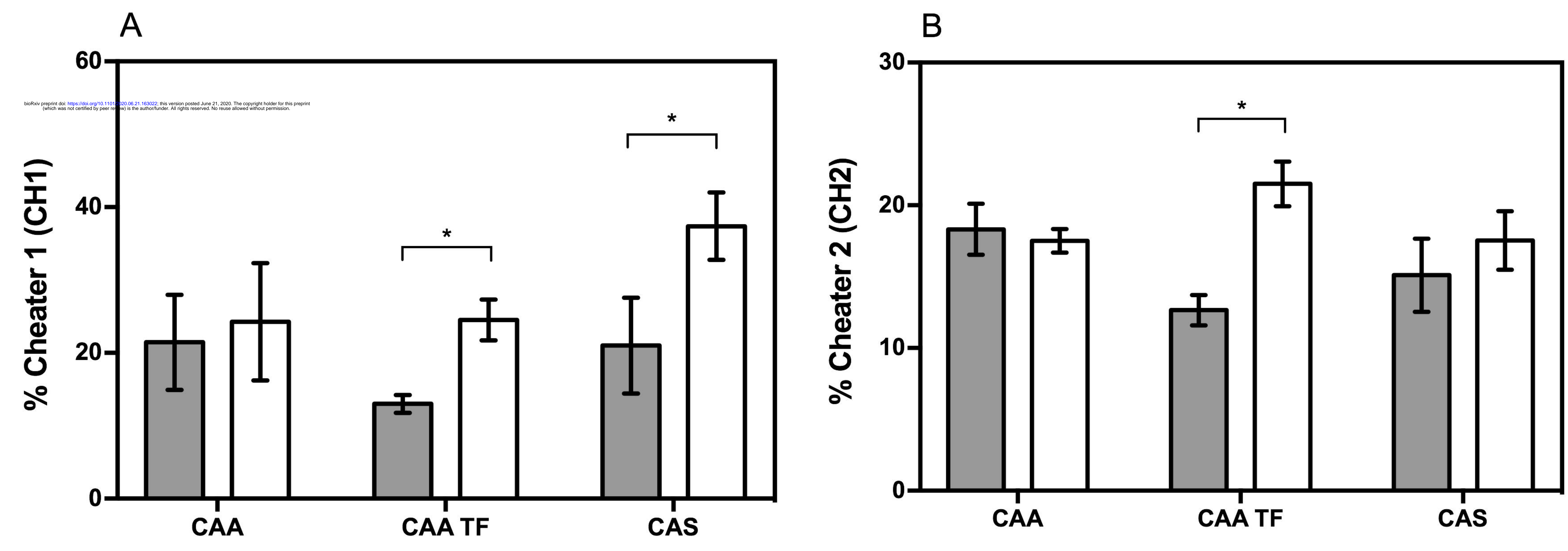


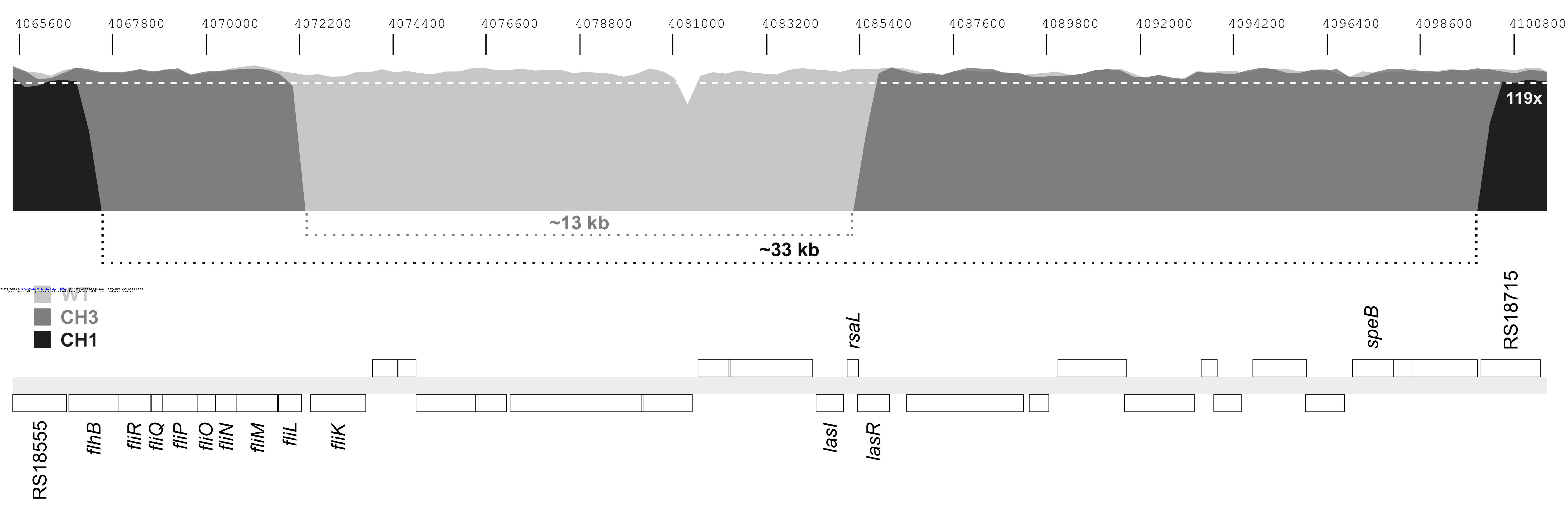




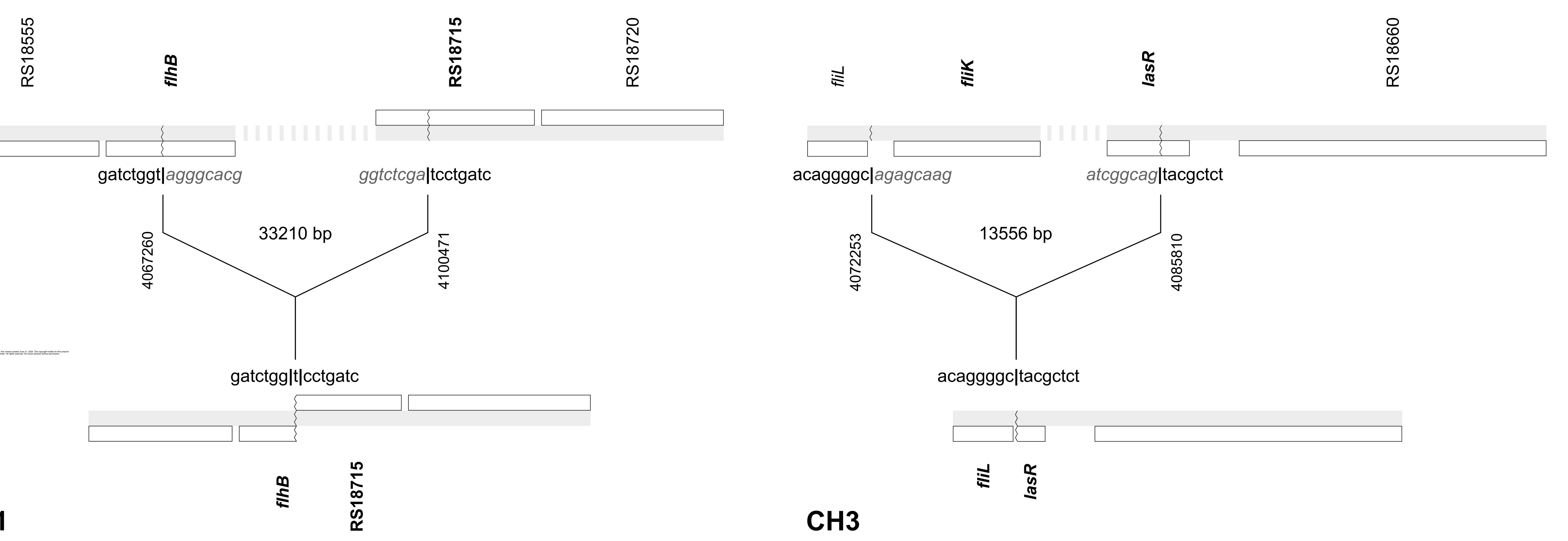

